\title{
Effects of Progressive Resistance Training on Post-Surgery Incontinence in Men with Prostate Cancer
}

\author{
Juhyun Park ${ }^{1,+}\left(\mathbb{D}\right.$, Dong Hyun Yoon ${ }^{2,+}{ }^{+}$, Sangjun Yoo ${ }^{1}$, Sung Yong Cho ${ }^{1}$, Min Chul Cho ${ }^{1}$, \\ Ga-Young Han ${ }^{2}$, Wook Song ${ }^{2,3, *}$ and Hyeon Jeong ${ }^{1, *}$ \\ 1 Department of Urology, Seoul National University College of Medicine, SMG-SNU Boramae Medical Center, \\ Seoul 07061, Korea; urojpark@naver.com (J.P.); ebend@naver.com (S.Y.); kmoretry@daum.net (S.Y.C.); \\ cmc1206@empal.com (M.C.C.) \\ 2 Department of Physical Education, Institute of Sport Science, Seoul National University College of \\ Education, Seoul 08826, Korea; ycool14@snu.ac.kr (D.H.Y.); 0104day@naver.com (G.-Y.H.) \\ 3 Institute on Aging, Seoul National University, Seoul 08826, Korea \\ * Correspondence: songw3@snu.ac.kr (W.S.); drjeongh@gmail.com (H.J.) \\ + These authors contributed equally to this work.
}

Received: 19 August 2018; Accepted: 18 September 2018; Published: 19 September 2018

\begin{abstract}
We evaluated the efficacy of progressive resistance training of the pelvic floor muscle for post-prostatectomy incontinence. In this prospective study, 59 patients who underwent radical prostatectomy were evaluated preoperatively. Continence was sequentially assessed within 2 weeks postoperatively, and an exercise regimen was initiated at 6- and 12-weeks. The primary outcome was continent status and the secondary outcome was changes in muscle strength and endurance after the exercise intervention. Continence was defined as no urine loss in a $1 \mathrm{~h}$ pad test. A total of 59 patients participated in this study. Six patients dropped out of the study because of non-compliance and orthopedic problems. Of the remaining 53 patients, 31 (58.5\%) achieved pad-free continence at 12 weeks postoperatively. The patients were divided into two groups based on their continence status, and no statistically significant difference was observed in age, body mass index, prostate volume, prostate-specific antigen, pathological Gleason score sum, and pathological T stage. Meanwhile, preoperative maximal urethral closure pressure and change in hip extensor muscle strength and endurance during the 12-week exercise program were significantly higher in the continent group. In multivariate analysis, change in hip extensor muscle strength was the only significant parameter predicting achievement of continence status (Odds ratio, 1.039; $p=0.045$ ). The changes in hip extensor muscle strength in the current exercise program was an independent predictor of continence status after radical prostatectomy. A large-scale prospective study on the relationship between extensor muscle strength and urinary incontinence should be explored in future.
\end{abstract}

Keywords: prostatectomy; incontinence; resistance training; pelvic floor muscle

\section{Introduction}

Prostate cancer is one of the most common cancers in Western countries [1]. In South Korea, the number of prostate cancer patients has increased also rapidly in recent decades, and the disease is now one of the top five most frequent cancers occurring in men in that country [2]. These dramatic changes in prostate cancer frequency may be attributed to early diagnosis, which became possible following introduction of the prostate-specific antigen (PSA) test. Consequently, the percentage of patients with advanced and metastatic prostate cancer has decreased while that of patients with 
early-stage localized prostate cancer has sharply increased [3,4]. Radical prostatectomy is recognized as a standard surgical treatment for early-stage localized prostate cancer [5].

Radical prostatectomy can be classified into open laparotomy and laparoscopic surgery using conventional laparoscopic instruments or robotic instruments. Each surgical method accesses the prostate in a different way [6]. However, the primary objective of these procedures is same to remove prostate, bilateral seminal vesicles, vas, and sufficient surrounding tissues to simultaneously avoid a positive resection margin and preserve the urethral sphincter and neurovascular bundle [6,7]. To date, several surgical techniques have been developed and applied to reduce urinary incontinence especially after radical prostatectomy [8].

Urinary incontinence can arise from damage during surgery to the urethral sphincter muscle or nerves controlling it $[9,10]$. Stress-type incontinence, a common type of incontinence that occurs during coughing or daily activities, can increase intra-abdominal pressure, and is difficult to cure with medical treatments $[10,11]$. Failing to address postoperative urinary incontinence could adversely affect patients' social and sexual lives, and act as a major factor that degrades health-related quality of life [11-13]. When post-prostatectomy incontinence is severe, it has been found to be associated with significantly lower patient satisfaction with the surgical treatment [13].

Kegel exercises are the most commonly practiced techniques for conservative management of post-prostatectomy incontinence [14], and various pelvic floor muscle exercise programs have been developed and spread widely for incontinence studies [15-17]. Those previous studies have not, however, investigated the mechanism of recovery from post-prostatectomy incontinence and have failed to make plausible assumptions [15-17]. There is a limit that the pelvic floor muscles is not a muscle that enters the surface and cannot be accurately measured. Thus, in current study, it was judgment that a movement to contraction an hip adductors, muscles of the pelvic region, muscles of the gluteal region, and abdominal muscles, called core muscle, could lead to stimulation and contraction in the pelvic floor muscles $[18,19]$.

We, therefore, evaluated the efficacy of a 12-week progressive resistance training program focused on pelvic floor muscles in improving recovery of continence after radical prostatectomy, and analyzed the changes in the exercise muscle strength and endurance in relation to continence status.

\section{Materials and Methods}

This prospective study was performed for whom received radical prostatectomy for prostate cancer in a single institute from January 2015 to April 2016. The inclusion criteria were: male patient aged 60 years or older, Eastern Cooperative Oncology Group performance status of 0 or 1, and written informed consents. Exclusion criteria were as follows: adjuvant or neoadjuvant chemo-radiation therapy, severe postoperative complications, history of pelvic surgery, and diseases that could affect voiding function and limitations for the exercise program such as for patients with serious cardiovascular events or spinal or articular disease. During the periods, single surgeon (HJ) performed the radical prostatectomy. The biopsy and prostatectomy specimens were assessed by well-experienced genitourinary pathologists. Clinicopathological data, including urodynamic study, were obtained. Multichannel video urodynamic study (MMS UD-2000, Medical Measurement System, Enscheded, The Netherlands) was performed twice: once at the preoperative visit and again at 12 weeks after starting of the exercise program [20]. Changes in the maximal urethral closure pressure (MUCP), maximal cystometric capacity and detrusor pressure at maximal urinary flow (PdetQmax) were also calculated.

The Institutional Review Board (IRB) approved this study protocol. The study conformed to the tenets of the Declaration of Helsinki. Personal patient identifiers were completely removed, and data were analyzed anonymously. 


\subsection{Pelvic Floor Muscle Exercise Program}

Progressive resistance training focused on pelvic floor muscles was initiated within 2 weeks postoperatively and conducted for 12 weeks thereafter (Figure 1). This intervention recommended that Kegel-based weight-bearing exercise be started at 0-6 weeks (phase 1) postoperatively, and then elastic-band-based high-speed power training until 12 weeks (phase 2). Each session included a 10-min warm-up, $40 \mathrm{~min}$ of Kegel-based exercise training and $10 \mathrm{~min}$ of cool-down. Resting periods of $1 \mathrm{~min}$ were allowed between sets and $2 \mathrm{~min}$ between exercises. High-speed resistance training is defined as a contraction phase expected to be accomplished as quickly as possible, with a 1-s pause and an eccentric contraction exceeding 2-s [21]. High-speed resistance exercise regimens are centered on the use of elastic exercise bands, based on a previous intervention [21]. The color of the band used served to define the exercise intensity. For the power training phase, green elastic bands (very low tension) were used and participants were instructed to exercise at a rate of perceived exertion of 12-13 ('somewhat hard'). Each high-speed resistance training exercise consisted of two to three sets of 10-12 repetitions. The exercise guideline and daily exercise checklist were created and distributed to the subjects, and the number of days of exercise was checked by bringing them in during their mid-term visits and the last visit. In addition, we performed different sets and repetitions to set individual exercise intensity. Afterwards, after an intermediate visit, the elastic band was used to increase the intensity of the exercise through tests. The exercise program followed the ACSM's Guide to Exercise and Cancer Survivorship [22].
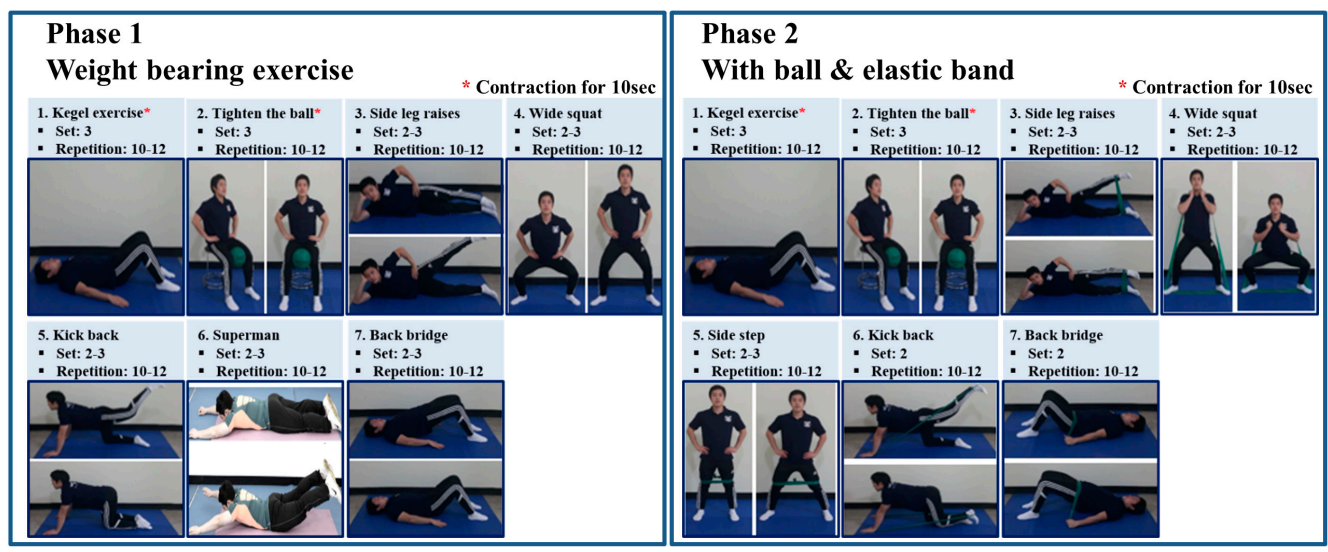

Figure 1. Exercise program protocol. ${ }^{*}$ Contraction for $10 \mathrm{~s}$.

\subsection{Lower Muscle Strength and Endurance}

Lower limb concentric dynamic strength was measured using a HUMAC NORM isokinetic dynamometer (CSMi Solutions, Stoughton MA, USA). The hip extension/flexion and abduction/adduction peak torques of each lower limb were evaluated for the isokinetic contraction test. The participants performed the test for a maximum of three or five repetitions. Each maximal strength test was performed with an angular speed of $60^{\circ} / \mathrm{s}$ (velocity of $60^{\circ} / \mathrm{s}$ ) for isokinetic muscle strength, and angular speed of $180^{\circ} / \mathrm{s}$ (velocity of $180^{\circ} / \mathrm{s}$ ) for isokinetic muscle endurance measurement. The exercise was performed twice prior to testing to obtain optimal results by allowing the participants to familiarize themselves with the test [21].

\subsection{Outcome Assessment}

The primary outcome was continence status and the secondary outcome was change in muscle strength and endurance after the exercise intervention. The severity of urinary incontinence was measured using a $1 \mathrm{~h}$ pad test [23]. "Urinary continence" was defined as no urine loss in a $1 \mathrm{~h}$ pad test. Incontinence and recovery were sequentially assessed within 2 weeks postoperatively, and at 6 and 12 weeks after initiation of the exercise program. 


\section{Statistical Analysis}

Results are presented as means \pm standard deviation. Statistically significant differences of the preoperative parameters in the subgroups were analyzed using analysis of variance, chi-square, independent $t$ tests and paired $t$ tests. Predictors of the 12-week continence status were evaluated using logistic regression analysis. The $p$-values were two-sided, with $p<0.05$ considered statistically significant. Statistical analysis was performed using SPSS ${ }^{\circledR}$ Version 22.0 (IBM, Armonk, NY, USA).

\section{Results}

From January 2015 to April 2016, a total of 72 patients underwent radical prostatectomy for treatment of prostate cancer at our institute. Of these, 69 were screened in accordance with the inclusion and exclusion criteria and deemed suitable candidates. The study was explained to the target group and 59 patients agreed to participate and provided their informed consent. The exercise program was initiated within 2 weeks postoperatively and was conducted for 12 weeks thereafter. Primary intermediate inspection was carried out after the first 6-week weight-bearing resistance exercise, and three patients dropped out. In the final 6-week elastic band resistance exercise, another three patients dropped out from the study. Ultimately, 53 patients completed follow-up (Figure 2). There was no adverse events related the exercise program.

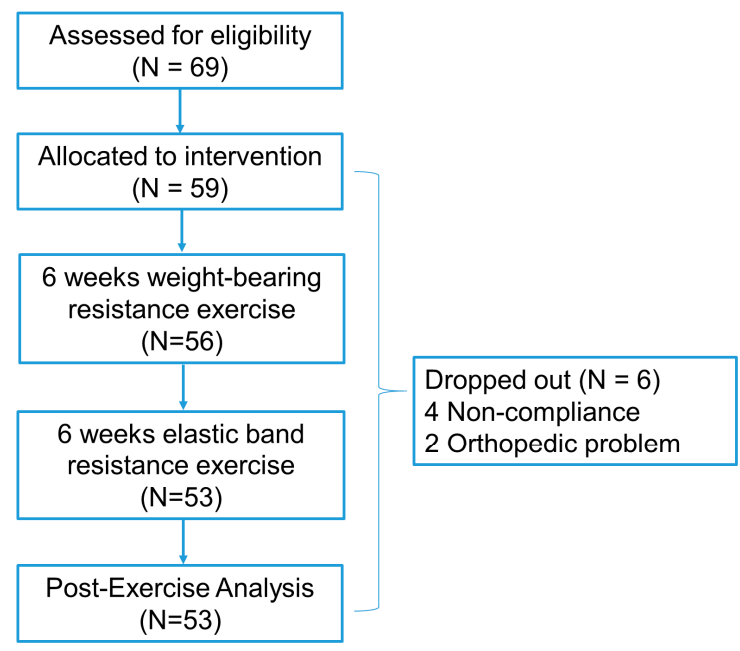

Figure 2. Study flow chart for 12-week exercise program.

In the final analysis, 58.5\% (31/53) of the patients achieved pad-free status after the 12-week exercise program. When divided into continent and incontinent groups based on pad-free status (continence), there was no difference in terms of age, body mass index (BMI), prostate volume, PSA, pathological Gleason score sum, and pathological T stage between the two groups. However, preoperative MUCP was significantly higher in the continent group, though postoperative MUCP after the 12-week exercise program did not significantly differ between the groups. Furthermore, there were no meaningful changes in MUCP during the exercise program. At the 6- and 12-week evaluations, the patients in the continent group used significantly fewer pads than those in the incontinent group (Table 1). 
Table 1. Patient characteristics according to the achievement of post-exercise continent status.

\begin{tabular}{|c|c|c|c|}
\hline & $\begin{array}{l}\text { Continent Group } \\
\qquad(n=31)\end{array}$ & $\begin{array}{l}\text { Incontinent Group } \\
\qquad(n=22)\end{array}$ & $p$-Value \\
\hline Age (years) & $68.2 \pm 6.1$ & $68.3 \pm 5.1$ & 0.899 \\
\hline Preoperative BMI $\left(\mathrm{kg} / \mathrm{m}^{2}\right)$ & $24.8 \pm 2.8$ & $26.6 \pm 9.0$ & 0.289 \\
\hline Post-exercise 12-weeks BMI $\left(\mathrm{kg} / \mathrm{m}^{2}\right)$ & $24.5 \pm 2.8$ & $24.7 \pm 3.3$ & 0.827 \\
\hline Prostate volume $(\mathrm{mL})$ & $36.4 \pm 19.1$ & $37.8 \pm 23.5$ & 0.827 \\
\hline PSA (ng/dL) & $23.6 \pm 42.1$ & $24.3 \pm 42.8$ & 0.953 \\
\hline Op methods & & & 0.601 \\
\hline RRP & 17 & 12 & \\
\hline RALP & 14 & 10 & \\
\hline Pathology Gleason Score Sum & & & 0.812 \\
\hline 6 & 5 & 4 & \\
\hline $7(3+4)$ & 7 & 5 & \\
\hline $7(4+3)$ & 8 & 8 & \\
\hline 8 & 7 & 4 & \\
\hline 9 & 4 & 1 & \\
\hline Pathologic T stage & & & 0.219 \\
\hline $\mathrm{T} 2$ & $20(64.5 \%)$ & $11(50.0 \%)$ & \\
\hline $\mathrm{T} 3$ & $11(35.5 \%)$ & $11(50.0 \%)$ & \\
\hline Participation rate $(\%)$ & $42.0 \pm 38.7$ & $50.4 \pm 36.0$ & 0.428 \\
\hline Preoperative MUCP $\left(\mathrm{cmH}_{2} \mathrm{O}\right)$ & $78.2 \pm 18.8$ & $64.8 \pm 25.3$ & 0.036 \\
\hline Preoperative MCC $(\mathrm{mL})$ & $355.3 \pm 133.1$ & $339.4 \pm 139.2$ & 0.685 \\
\hline Preoperative PdetQmax $\left(\mathrm{cm} \mathrm{H}_{2} \mathrm{O}\right)$ & $55.1 \pm 21.0$ & $48.7 \pm 20.2$ & 0.285 \\
\hline Post-exercise 12-week MUCP $\left(\mathrm{cm} \mathrm{H}_{2} \mathrm{O}\right)$ & $56.1 \pm 25.3$ & $53.6 \pm 27.0$ & 0.739 \\
\hline Post-exercise 12-week MCC $(\mathrm{mL})$ & $339.5 \pm 89.7$ & $297.1 \pm 77.3$ & 0.085 \\
\hline Post-exercise 12-week DetQmax $\left(\mathrm{cm} \mathrm{H}_{2} \mathrm{O}\right)$ & $38.5 \pm 18.5$ & $30.6 \pm 15.6$ & 0.123 \\
\hline Change of MUCP $\left(\mathrm{cm} \mathrm{H}_{2} \mathrm{O}\right)$ & $24.4 \pm 29.4$ & $12.9 \pm 30.6$ & 0.199 \\
\hline Post-exercise 6-week pad (g) & $14.2 \pm 45.6$ & $18.7 \pm 23.8$ & 0.676 \\
\hline Post-exercise 6-week pad number & $1.67 \pm 2.1$ & $4.2 \pm 5.8$ & 0.034 \\
\hline Post-exercise 12-week pad (g) & & $14.7 \pm 25.1$ & 0.002 \\
\hline Post-exercise 12-week pad number & $0.4 \pm 0.5$ & $2.14 \pm 2.4$ & $<0.001$ \\
\hline
\end{tabular}

BMI, Body mass index; PSA, prostate-specific antigen; RRP, retropubic radial prostatectomy; RALP, robot-assisted laparoscopic radical prostatectomy; MUCP, Maximal urethra closure pressure; MCC, maximal cystometric capacity; PdetQmax, detrusor pressure at maximal urinary flow in pressure-flow study.

Changes in hip extensor muscle strength and endurance during the 12-week exercise program were significantly higher in the continent group than the incontinent group, whereas the measured values of each participant's lower limb muscle strength and endurance were comparable between the groups (Table 2). 
Table 2. Change of pelvic muscle strength and endurance according to the achievement of post-exercise continent status.

\begin{tabular}{|c|c|c|c|c|c|}
\hline & \multicolumn{2}{|c|}{$\begin{array}{l}\text { Continent Group } \\
\qquad(n=31)\end{array}$} & \multicolumn{2}{|c|}{$\begin{array}{l}\text { Incontinent Group } \\
\qquad(n=22)\end{array}$} & \multirow[t]{2}{*}{$p$-Value } \\
\hline & Pre & Post & Pre & Post & \\
\hline \multicolumn{6}{|c|}{ Isokinetic $60^{\circ} / \mathrm{s}$ peak torque/Bodyweight, muscle strength } \\
\hline \multicolumn{6}{|c|}{ Hip Extensor $(\mathrm{Nm})$} \\
\hline Pre/Post exercise muscle strength & $119.5+41.7$ & $145.9+41.3$ & $138.0+67.5$ & $139.6+55.4$ & \\
\hline Change of muscle strength & \multicolumn{2}{|c|}{$22.3 \pm 39.7$} & \multicolumn{2}{|c|}{$-9.5 \pm 32.4$} & 0.005 \\
\hline $\begin{array}{l}\text { Hip Flexor (Nm) } \\
\text { Pre/Post exercise muscle strength }\end{array}$ & $78.1+25.9$ & $92.2+26.1$ & $85.0+$ & $90.6+19.3$ & \\
\hline Change of muscle strength & \multirow{2}{*}{\multicolumn{2}{|c|}{$11.5 \pm 26.9$}} & \multirow{2}{*}{\multicolumn{2}{|c|}{$1.4 \pm 27.2$}} & 0.204 \\
\hline Hip Abductor $(\mathrm{Nm})$ & & & & & \\
\hline Pre/Post exercise muscle strength & $75.5+19.4$ & $87.5+28.2$ & $70.5+24.7$ & $71.9+32.7$ & \\
\hline $\begin{array}{l}\text { Change of muscle strength } \\
\text { Hip Adductor (Nm) }\end{array}$ & \multicolumn{2}{|c|}{$12.0 \pm 26.4$} & \multicolumn{2}{|c|}{$10.2 \pm 26.3$} & 0.811 \\
\hline Pre/Post exercise muscle strength & $109.1+37.7$ & $122.2+40.5$ & $116.1+43.5$ & $130.7+41.5$ & \\
\hline Change of muscle strength & \multicolumn{2}{|c|}{$21.7 \pm 48.4$} & \multicolumn{2}{|c|}{$3.9 \pm 31.0$} & 0.153 \\
\hline \multicolumn{6}{|c|}{$\begin{array}{l}\text { Isokinetic } 180^{\circ} / \mathrm{s} \text { peak torque / Bodyweight, muscle endurance } \\
\text { Hip Extensor (Nm) }\end{array}$} \\
\hline Pre/Post exercise muscle endurance & $85.3+65.4$ & $106.9+38.8$ & $102.5+53.9$ & $105.1+42.6$ & \\
\hline $\begin{array}{l}\text { Change of muscle endurance } \\
\text { Hip Flexor (Nm) }\end{array}$ & \multicolumn{2}{|c|}{$18.8 \pm 43.6$} & \multicolumn{2}{|c|}{$-5.6 \pm 33.1$} & 0.038 \\
\hline Pre/Post exercise muscle endurance & $65.1+65.4$ & $62.1+17.4$ & $58.8+25.2$ & $66.5+18.1$ & \\
\hline $\begin{array}{l}\text { Change of muscle endurance } \\
\text { Hip Abductor (Nm) }\end{array}$ & \multicolumn{2}{|c|}{$-5.2 \pm 61.9$} & \multicolumn{2}{|c|}{$4.2 \pm 20.1$} & 0.517 \\
\hline Pre/Post exercise muscle endurance & $63.0+21.0$ & $70.9+24.0$ & $64.8+23.0$ & $66.5+18.1$ & \\
\hline $\begin{array}{l}\text { Change of muscle endurance } \\
\text { Hip Adductor (Nm) }\end{array}$ & \multicolumn{2}{|c|}{$7.9 \pm 25.6$} & \multicolumn{2}{|c|}{$3.1 \pm 14.3$} & 0.449 \\
\hline Pre/Post exercise muscle endurance & $79.3+36.3$ & $89.3+29.9$ & $82.4+30.4$ & $89.1+36.2$ & \\
\hline Change of muscle endurance & \multicolumn{2}{|c|}{$10.0 \pm 32.2$} & \multicolumn{2}{|c|}{$5.7 \pm 27.7$} & 0.624 \\
\hline
\end{tabular}

In univariate analysis, the preoperative MUCP (odds ratio, $1.031 ; p=0.045$ ) and changes in the hip extensor muscle strength during the exercise program (odds ratio, 1.029; $p=0.010$ ) were found to be significant variables for forecasting continence status after the 12-week exercise program. In multivariate analysis, hip extensor muscle strength was the only significant parameter predicting continence status (odds ratio, 1.039; $p=0.045$; Table 3).

Table 3. Logistic regression analysis to predict post-exercise 12 week continent status.

\begin{tabular}{ccccc}
\hline & \multicolumn{2}{c}{ Univariate } & \multicolumn{2}{c}{ Multivariate } \\
\cline { 2 - 5 } & $p$-Value & OR (95\% CI) & $p$ Value & OR (95\% CI) \\
\hline Age & 0.897 & $0.994(0.901-1.096)$ & 0.686 & $0.965(0.811-1.148)$ \\
BMI & 0.354 & $0.943(0.833-1.067)$ & 0.800 & $0.963(0.719-1.289)$ \\
Prostate volume & 0.822 & $0.997(0.968-1.026)$ & 0.454 & $0.984(0.942-1.027)$ \\
Pathologic T stage & 0.293 & $0.550(0.181-1.675)$ & 0.773 & $1.369(0.162-11.535)$ \\
Operative Methods & 0.983 & $0.988(0.330-2.962)$ & 0.553 & $1.811(0.280-11.703)$ \\
(RRP vs. RALP) & 0.045 & $1.031(1.001-1.061)$ & 0.097 & $1.046(0.992-1.103)$ \\
MUCP & 0.010 & $1.029(1.007-1.053)$ & 0.045 & $1.039(1.001-1.080)$ \\
\hline
\end{tabular}

BMI, Body mass index; RRP, retropubic radial prostatectomy; RALP, robot-assisted laparoscopic radical prostatectomy; MUCP, Maximal urethra closure pressure.

\section{Discussion}

Urinary incontinence is a major problem for men undergoing prostatectomy for treatment of their cancer, and leads to considerably reduced quality of life [12]. Therapies to reduce the incidence 
and severity of incontinence in these patients need to be developed and researched with regard to their efficacy. Exercise medicine interventions are particularly attractive given their relatively rapid and inexpensive implementation, and lack of side effects such as those inherent in any pharmaceutical approach $[10,24]$. Therefore, a recently published study protocol was planned using different times of exercise initiation, exercise durations, and combinations of exercise programs [15-17]. Centemero and his colleagues published the early starting of pelvic floor muscle exercise before radical prostatectomy could improve early continence. In that study, physiotherapist helped the pelvic floor muscle exercise twice per week [15]. Parekh et al revealed the formal education and instruction of pelvic floor muscle exercise showed significant benefit to achieve continent status [16]. Tienforti et al. showed that preoperative biofeedback combined with a postoperative pelvic floor muscle exercise would be more effective treatment strategy in improving recovering of incontinence [17].

Thus far, previous studies have not investigated in detail the mechanism how pelvic floor muscle exercises can help patients recover from urinary incontinence [15-17]. Because the pelvic floor muscles could not be accurately measured and specifically trained, lower limb exercise of current exercise program to contract an hip adductors, muscles of the pelvic region, muscles of the gluteal region, and abdominal muscles might be the clues for the recovering mechanism from post-prostatectomy incontinence $[18,19]$. The changes of hip joint muscle strength or endurance were not simply the change of pelvic floor muscle strength or endurance. However, the continent group, the change of hip extensor muscle strength and endurance were definitely significant between the continent and incontinent groups. Ultimately, it could be reasonable to hypothesize that the change in hip extensor muscle strength is related to achievement of continence status and to design another exercise program to focus the reinforcement of hip extensor muscle.

Interestingly, in the present study, the rate of achieving continence did not differ by degree of participation in exercise therapy. When patients were classified into an active and a passive group according to their degree of participation in the training program based on a threshold of $50 \%$ participation, 27 patients were classified into the former and 26 in the latter. Contrary to expectations, the rates of continence in the active and passive groups were $55.6 \%$ and $61.5 \%$, respectively, and the difference was not statistically significant. These results suggested that performing the exercise correctly may be more important in strengthening the essential muscles associated with recovery from urinary incontinence.

This study was the first to reveal that change in hip extensor muscle strength and endurance could be related to achievement of continence, and this novel finding can be applied clinically in various ways. First, a newly developed exercise method can be provided to reinforce hip extensor muscle in patients with incontinence after radical prostatectomy. Furthermore, a new exercise method can be adopted to treat stress urinary incontinence in women and incontinence in men after transurethral prostatectomy such as holmium laser enucleation of the prostate [25].

In recent reviews, pelvic floor muscle exercise for urinary incontinence after radical prostatectomy was considered an effective treatment option for accelerating restoration of continence [9-11]. Similarly, preoperative pelvic floor muscle exercises were reported to potentially aid faster return to continent status at 3 months postoperatively, though there were no significant differences in continence rates at 6 months postoperatively [24]. Although there was no difference in the long-term continence rates, the results remain meaningful if the exercise program enables faster recovery from urinary incontinence. Early recovery from urinary incontinence is also central issue for patients who undergo prostatectomy $[11,24,26]$.

The severity of urinary incontinence was measured using a $1 \mathrm{~h}$ pad test, which was standardized as the specific protocol for evaluating urinary incontinence [23]. Other pelvic floor muscle exercise studies assessed the degree of incontinence based on a $24 \mathrm{~h}$ pad test or the number of pads used in a day [15-17]. In present study, self-reported numbers of pads used per day was not matched with continent status. Some patients with a negative $1 \mathrm{~h}$ pad test used pads in real life, while other patients 
with a positive $1 \mathrm{~h}$ pad test used no pads. The patient's individual character influence the number of pads used daily.

Some studies have tried to improve recovery from incontinence by using a meticulous minimally invasive technique to preserve the neurovascular bundles during surgery [26]. Recovery of urinary incontinence after radical prostatectomy is also expected to be faster if applying posterior musculofascial reconstruction during the surgery [27]. In the present study, a single surgeon $(\mathrm{HJ})$ performed all the radical prostatectomy procedures. Two operative methods were used: robot-assisted laparoscopic radical prostatectomy and retropubic radical prostatectomy. Posterior reconstruction was performed during each surgical technique. The rate of continence achievement was not related to the surgical method used.

The present study showed that appropriate exercise intervention after radical prostatectomy in patients with prostate cancer has great merit for recovery of general physical and mental condition [11-13]. The study does have some limitations. For one, it was a prospective study, but it was not a randomized controlled study. Additionally, the sample size of patients was relatively small, and $10 \%$ of the initial study population stopped participating. Finally, a single operator performed the radical prostatectomy, but two different surgical methods were applied.

Nevertheless, the results of the present study are meaningful, as we reported the effect of pelvic exercise therapy on change in hip joint muscle strength and found the exercise program yielded significant results. Future studies on urinary incontinence should develop more structured and efficient exercise methods that can reinforce hip extensor muscle.

\section{Conclusions}

The total continence rate in patients who underwent radical prostatectomy was $58.5 \%$ following a supervisor-assisted 12-week progressive resistance training program. Change in hip extensor muscle strength during the exercise program was an independent predictor of post-prostatectomy continence status. The relationship between hip extensor muscle and urinary incontinence should be explored in future studies.

Author Contributions: Conceptualization, J.P., S.Y., S.Y.C., M.C.C., G.Y.-H., D.H.Y., W.S. and H.J.; Methodology, J.P., S.Y., S.Y.C., M.C.C., G.Y.-H., D.H.Y., W.S. and H.J.; Project administration, J.P., S.Y., S.Y.C., M.C.C. and H.J.; Funding acquisition, H.J.; Data curation, J.P., S.Y., G.Y.-H. and D.H.Y.; Formal analysis, J.P., M.C.C., G.Y.-H., D.H.Y., W.S. and H.J.; Investigation, J.P., G.Y.-H., D.H.Y. and H.J.; Development, J.P.; Writing-original draft, J.P. and D.H.Y.; Resources, S.Y.C., M.C.C., G.Y.-H., D.H.Y. and W.S.; Writing-review \& editing, M.C.C., W.S. and H.J.; Supervision, W.S. and H.J.

Funding: This work was supported by a clinical research grant-in-aid from the Seoul Metropolitan Government Seoul National University (SMG-SNU) Boramae Medical Center (02-2016-4).

Conflicts of Interest: Nothing to declare.

\section{References}

1. Siegel, R.L.; Miller, K.D.; Jemal, A. Cancer statistics. Cancer J. Clin. 2017, 67, 7-30. [CrossRef] [PubMed]

2. Jung, K.W.; Won, Y.J.; Kong, H.J.; Oh, C.M.; Cho, H.; Lee, D.H.; Lee, K.H. Cancer statistics in Korea: Incidence, mortality, survival, and prevalence in 2012. Cancer Res. Treat. 2015, 47, 127-141. [CrossRef] [PubMed]

3. Heo, J.E.; Ahn, H.K.; Kim, J.; Chung, B.H.; Lee, K.S. Changes in clinical characteristics of patients with an initial diagnosis of prostate cancer in Korea: 10-year trends reported by a tertiary center. J. Korean Med. Sci. 2018, 33. [CrossRef] [PubMed]

4. Kim, D.; Choi, D.; Lim, J.H.; Yoon, J.H.; Jeong, I.G.; You, D.; Hong, J.H.; Ahn, H.; Kim, C.S. Changes in prostate cancer aggressiveness over a 12-year period in Korea. Korean J. Urol. 2012, 53, 680-685. [CrossRef] [PubMed]

5. Singh, J.; Trabulsi, E.J.; Gomella, L.G. Is there an optimal management for localized prostate cancer? Clin. Interv. Aging 2010, 5, 187-197. [PubMed] 
6. Ilic, D.; Evans, S.M.; Allan, C.A.; Jung, J.H.; Murphy, D.; Frydenberg, M. Laparoscopic and robot-assisted vs. open radical prostatectomy for the treatment of localized prostate cancer: A cochrane systematic review. BJU Int. 2017, 6, 845-853. [CrossRef] [PubMed]

7. Borregales, L.D.; Berg, W.T.; Tal, O.; Wambi, C.; Kaufman, S.; Gaya, J.M.; Urzua, C.; Badani, K.K. 'Trifecta' after radical prostatectomy: Is there a standard definition? BJU Int. 2013, 112, 60-67. [CrossRef] [PubMed]

8. Manfredi, M.; Fiori, C.; Amparore, D.; Checcucci, E.; Porpiglia, F. Technical details to achieve perfect early continence after radical prostatectomy. Minerva Chir. 2018. [CrossRef]

9. Kretschmer, A.; Hubner, W.; Sandhu, J.S.; Bauer, R.M. Evaluation and management of postprostatectomy incontinence: A systematic review of current literature. Eur. Urol. Focus 2016, 2, 245-259. [CrossRef] [PubMed]

10. Radadia, K.D.; Farber, N.J.; Shinder, B.; Polotti, C.F.; Milas, L.J.; Tunuguntla, H. Management of postradical prostatectomy urinary incontinence: A review. Urology 2017, 113, 13-19. [CrossRef] [PubMed]

11. Newman, D.K.; Guzzo, T.; Lee, D.; Jayadevappa, R. An evidence-based strategy for the conservative management of the male patient with incontinence. Curr. Opin. Urol. 2014, 24, 553-559. [CrossRef] [PubMed]

12. Trofimenko, V.; Myers, J.B.; Brant, W.O. Post-prostatectomy incontinence: How common and bothersome is it really? Sex. Med. Rev. 2017, 5, 536-543. [CrossRef] [PubMed]

13. Baker, H.; Wellman, S.; Lavender, V. Functional quality-of-life outcomes reported by men treated for localized prostate cancer: A systematic literature review. Oncol. Nurs. Forum 2016, 43, 199-218. [CrossRef] [PubMed]

14. Bridgeman, B.; Roberts, S.G. The 4-3-2 method for kegel exercises. Am. J. Men's Health 2010, 4, 75-76. [CrossRef] [PubMed]

15. Centemero, A.; Rigatti, L.; Giraudo, D.; Lazzeri, M.; Lughezzani, G.; Zugna, D.; Montorsi, F.; Rigatti, P.; Guazzoni, G. Preoperative pelvic floor muscle exercise for early continence after radical prostatectomy: A randomised controlled study. Eur. Urol. 2010, 57, 1039-1043. [CrossRef] [PubMed]

16. Parekh, A.R.; Feng, M.I.; Kirages, D.; Bremner, H.; Kaswick, J.; Aboseif, S. The role of pelvic floor exercises on post-prostatectomy incontinence. J. Urol. 2003, 170, 130-133. [CrossRef] [PubMed]

17. Tienforti, D.; Sacco, E.; Marangi, F.; D’Addessi, A.; Racioppi, M.; Gulino, G.; Pinto, F.; Totaro, A.; D’Agostino, D.; Bassi, P. Efficacy of an assisted low-intensity programme of perioperative pelvic floor muscle training in improving the recovery of continence after radical prostatectomy: A randomized controlled trial. BJU Int. 2012, 110, 1004-1010. [CrossRef] [PubMed]

18. Di Benedetto, P.; Coidessa, A.; Floris, S. Rationale of pelvic floor muscles training in women with urinary incontinence. Miner. Ginecol. 2008, 60, 529-541.

19. Martuscello, J.M.; Nuzzo, J.L.; Ashley, C.D.; Campbell, B.I.; Orriola, J.J.; Mayer, J.M. Systematic review of core muscle activity during physical fitness exercises. J. Strength Cond. Res. 2013, 27, 1684-1698. [CrossRef] [PubMed]

20. Rosier, P.; Schaefer, W.; Lose, G.; Goldman, H.B.; Guralnick, M.; Eustice, S.; Dickinson, T.; Hashim, H. International continence society good urodynamic practices and terms 2016: Urodynamics, uroflowmetry, cystometry, and pressure-flow study. Neurourol. Urodyn. 2017, 36, 1243-1260. [CrossRef] [PubMed]

21. Yoon, D.H.; Kang, D.; Kim, H.J.; Kim, J.S.; Song, H.S.; Song, W. Effect of elastic band-based high-speed power training on cognitive function, physical performance and muscle strength in older women with mild cognitive impairment. Geriatr. Gerontol. Int. 2017, 17, 765-772. [CrossRef] [PubMed]

22. Irwin, M.L.; American College of Sports Medicine. Acsm's Guide to Exercise and Cancer Survivorship; Human Kinetics: Champaign, IL, USA, 2012.

23. Krhut, J.; Zachoval, R.; Smith, P.P.; Rosier, P.F.; Valansky, L.; Martan, A.; Zvara, P. Pad weight testing in the evaluation of urinary incontinence. Neurourol. Urodyn. 2014, 33, 507-510. [CrossRef] [PubMed]

24. Chang, J.I.; Lam, V.; Patel, M.I. Preoperative pelvic floor muscle exercise and postprostatectomy incontinence: A systematic review and meta-analysis. Eur. Urol. 2016, 69, 460-467. [CrossRef] [PubMed]

25. Lee, Y.J.; Oh, S.A.; Kim, S.H.; Oh, S.-J. Patient satisfaction after holmium laser enucleation of the prostate (holep): A prospective cohort study. PLoS ONE 2017, 12, e0182230. [CrossRef] [PubMed] 
26. Reeves, F.; Preece, P.; Kapoor, J.; Everaerts, W.; Murphy, D.G.; Corcoran, N.M.; Costello, A.J. Preservation of the neurovascular bundles is associated with improved time to continence after radical prostatectomy but not long-term continence rates: Results of a systematic review and meta-analysis. Eur. Urol. 2015, 68, 692-704. [CrossRef] [PubMed]

27. Grasso, A.A.; Mistretta, F.A.; Sandri, M.; Cozzi, G.; De Lorenzis, E.; Rosso, M.; Albo, G.; Palmisano, F.; Mottrie, A.; Haese, A.; et al. Posterior musculofascial reconstruction after radical prostatectomy: An updated systematic review and a meta-analysis. BJU Int. 2016, 118, 20-34. [CrossRef] [PubMed]

(C) 2018 by the authors. Licensee MDPI, Basel, Switzerland. This article is an open access article distributed under the terms and conditions of the Creative Commons Attribution (CC BY) license (http:/ / creativecommons.org/licenses/by/4.0/). 